\title{
Downlink and Uplink Physical Channels in Long Term Evolution
}

\author{
A. Z. Yonis \\ College of Electronic Engineering, University of Mosul, Mosul, Iraq \\ Email: aws_zuher@yahoo.com
}

M. F. L. Abdullah

Faculty of Electrical and Electronic Engineering, University Tun Hus sein Onn Malaysia, Johor, Malaysia

Email: faiz@uthm.edu.my

\begin{abstract}
Long Term Evolution (LTE) defines a number of physical channels to carry information blocks received from the MAC and higher layers. This paper presents two types of Physical channels: the first type is downlink physical channels which consist of Physical Broadcast Channel (PBCH), Physical Downlink Shared Channel (PDSCH), Physical Multicast Channel (PMCH), Physical Do wnlink Control Channel (PDCCH), Physical Control Format Indicator Channel (PCFICH) and Physical Hybrid A RQ Indicator Channel (PHICH). The second type of Physical channels is uplink physical channels which consist of Physical Uplink Shared Channel (PUSCH), Physical Uplink Control Channel (PUCCH) and Physical Random Access Channel (PRACH). This paper also highlights the structure of PDSCH and $\mathrm{PUSCH}$, discuss the algorithms of the two types of physical channel and each of its features. The aim of this paper is to discuss the well-designed PHY Channels which provide high cell-edge performance with specific features, such as dynamic bandwidth allocation to users, the design of reference signals and control channels. These channels take into account a more challenging path loss and interference environment at the cell edge.
\end{abstract}

Index Terms - LTE, Downlink Physical Channel, Uplink Physical Channel

\section{Introduction}

In this paper the physical layer of LTE is described, based on the use of Orthogonal Frequency Division Multiple Access (OFDMA) and Single Carrier Frequency Division Multiple Access (SC-FDMA) principles as covered in this paper. The LTE physical layer is characterized by the design principle of resource usage based solely on dynamically allocated shared resources rather than having dedicated resources reserved for a single user.

The physical layer of a radio access system has a key role in defining the resulting capacity and becomes a focal point when comparing different systems for expected performance. A competitive system requires an efficient protocol layer to ensure good performance through both the application layer and the end user. This also enables the dynamic nature of the radio interface because all radio resource control is located close to the radio in the base station site. The 3rd Generation Partnership Project (3GPP) term for the base station used is eNodeB (different to the Wideband Code Division Multiple Access (WCDMA) BTS term, which is NodeB; e stands for 'evolved'). Therefore this paper initial covers the physical channel structures and then introduces the channel coding and physical layer procedures [1,2].

The paper will provide a more detailed description of the downlink/uplink physical-layer functionality, including the LTE channel types (section 2), details on downlink physical channels (Section 3). Also the paper will provide a corresponding description for the uplink physical layer (section 4, also the paper will then go further into the details of some communication channel structure (section5).

Finally it concludes with a description of physical layer measurements and device capabilities as well as a brief look at the aspects of the parameter configuration of the physical layer. In 3GPP specifications the physical layer covered in 36.2 series, with the four key physical layer specifications.

\section{LTE Channel Types}

There are three categories into which the various data channels may be grouped.

- Physical channels: These are transmission channels that carry user data and control messages.

- Transport channels: The physical layer transport channels offer information transfer to Medium Access Control (MAC) and higher layers. 
- Logical channels: Provide services for the Medium Access Control (MAC) layer within the LTE protocol structure.

This paper presents the physical channels for both types of downlink physical channels and uplink physical channels plus highlight on the PDSCH and PUSCH.

\section{Downlink Physical Channels}

LTE defines a nu mber of down link physical channels to carry information blocks received from the MAC and higher layers. These channels are categorized as transport or control channels as Fig. 1 shows radio architecture.

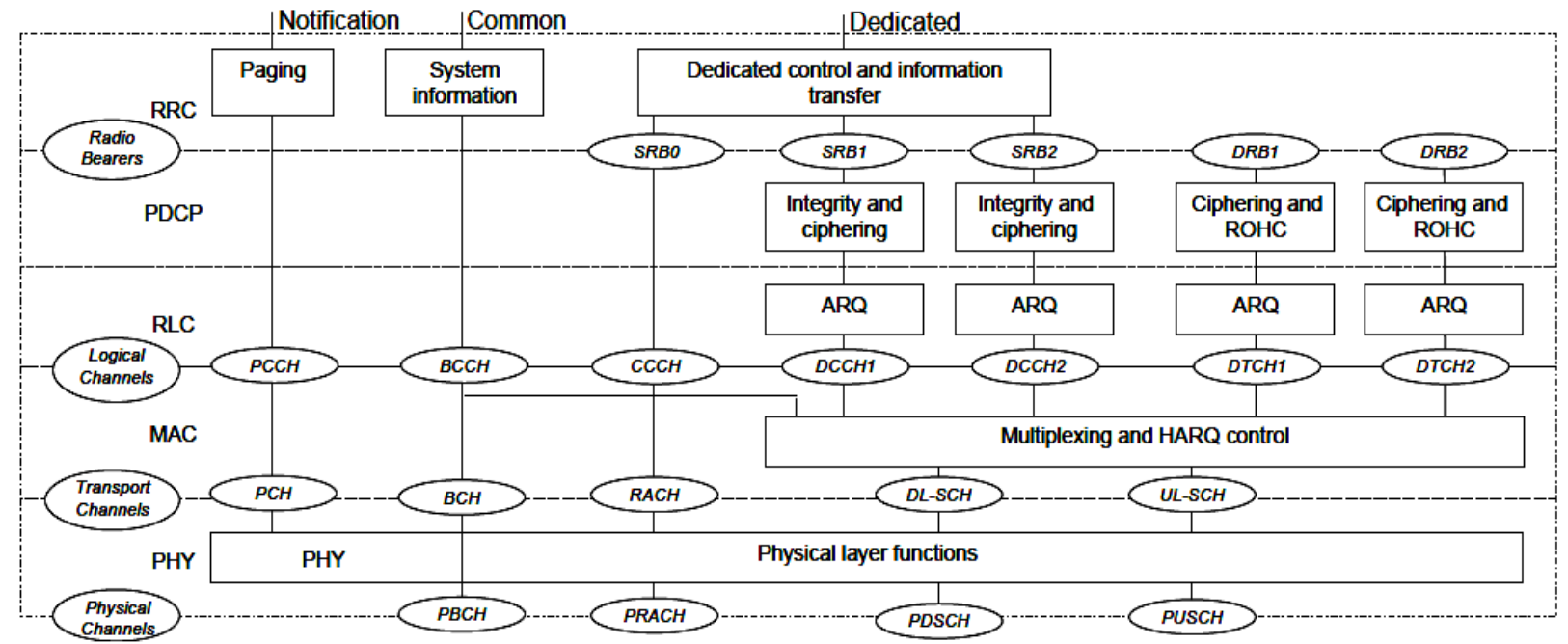

Fig. 1: Radio architecture

\subsection{Transport Channels}

\subsubsection{Physical Broadcast Channel (PBCH)}

The PBCH carries the MIB (Master Information Block) and has a payload of only 24 bits. The MIB informs DL bandwidth, the frame number and the PHICH configuration. Additionally, DL antenna configuration, $40 \mathrm{~ms}$ timing and the TX diversity type used for PBCH are also informed. Fig. 2 shows antenna port reference signal allocation.

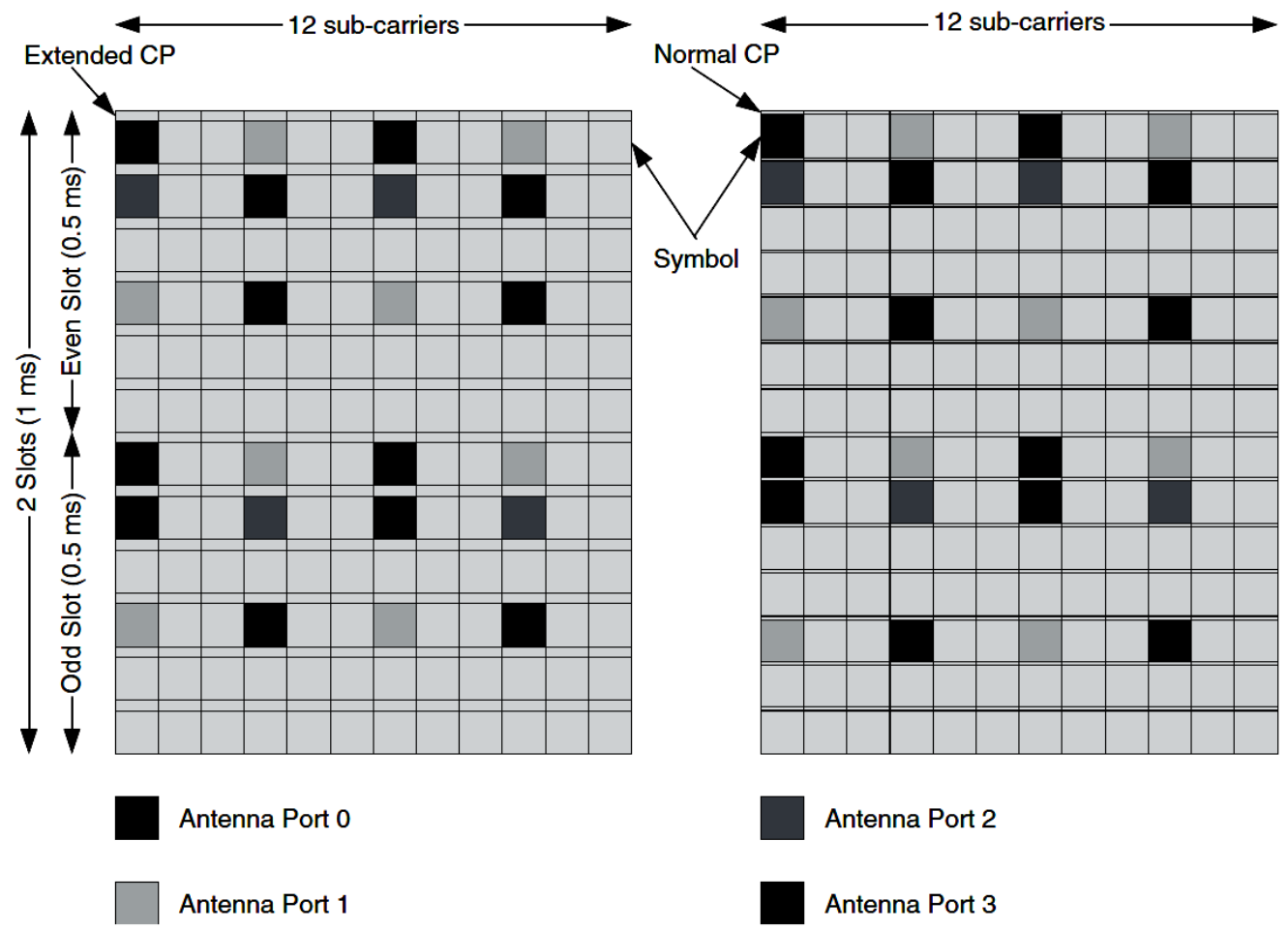

Fig. 2: Antenna port reference signal allocation 
This channel information is essential for decoding the remaining of the frame; therefore it is transmitted in a highly reliable form. A 16 bit cyclic redundancy check (CRC) is added to the payload and a 1/3 convolutional code is then applied, resulting in a total of $120 \mathrm{bits}$. The modulation technique used is quaternary phase shift keying (QPSK), resulting in 60 symbols.

The channel has assigned the first four symbols of slot 1 on 72 sub-carriers centered on the carrier frequency ( 4 sets of 6 resource blocks (RBs)). This implies that the information is always available at the same location regardless of the bandwidth. The channel information fits into one set of RBs, so that it has a repetition of 4 times per frame. The PBCH TTI (Transmission Time Interval) is $40 \mathrm{~ms}$, therefore the channel is repeated again in the three following frames, to a total of 16 repetitions (12 $\mathrm{dB}$ gain)[3].

\subsubsection{Physical Downlink Shared Channel (PDSCH)}

The PDSCH is the main data bearing channel which is allocated to the users on a dynamic and opportunistic basis. The PDSCH carries data in what's known as Transport Blocks (TB) which correspond to a MAC PDU. They are passed from the MAC layer to the PHY layer once per TTI which is $1 \mathrm{~ms}$ (i.e. $1 \mathrm{~ms}$ scheduling interval to meet low latency requirements).
In order to guard against propagation channel errors, convolutional turbo coder is used for forward error correction. The data is mapped to spatial layers according to the type of multi-antenna technique (e.g. closed loop spatial multiplexing, open-loop, spatial multip lexing, trans mit diversity, etc.) and then mapped to a modulation symbol which includes QPSK, 16 Quadrature amplitude modulation (QAM) and 64 QAM. Physical resources are assigned on a basis of two resource blocks for one TTI $(1 \mathrm{~ms})$. This is referred to as 'pair of resource blocks' which is the quantum of resources that can be allocated. It corresponds to 12 sub-carriers $(180 \mathrm{kHz})$ for 14 OFDM symbols (normal cyclic prefix (CP) mode).

The PDCH is also used to transmit broadcast information but not to transmit on the $\mathrm{PBCH}$ which include System Information Blocks (SIB) and paging messages.

The different steps of the DL-SCH physical layer processing are outlined in Fig. 3. In the case of carrier aggregation - that is, transmission on multiple component carriers in parallel to the same terminal of the transmissions on the different carriers correspond to separate transport channels with separate and more or less independent physical-layer processing. The transport-channel processing is outlined in Fig. 3 and the discussion below is thus valid also in the case of carrier aggregation.

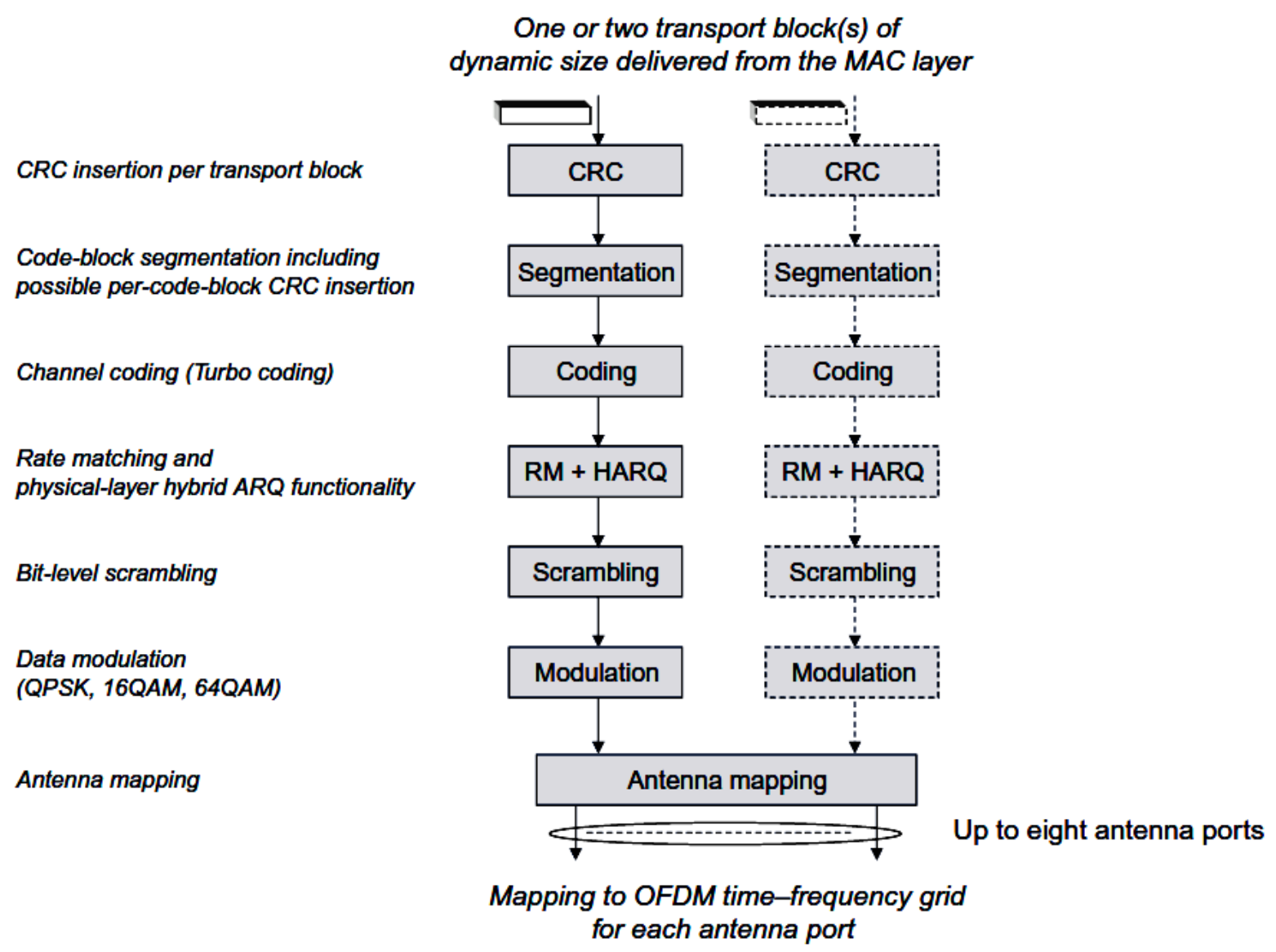

Fig. 3: Physical layer processing for DL-SCH [4] 
Within each Transmission Time Interval (TTI), corresponding to one subframe of length $1 \mathrm{~ms}$, up to two transport blocks of dynamic size are delivered to the physical layer and transmitted over the radio interface for each component carrier. The number of transport blocks transmitted within a TTI depends on the configuration of the multi-antenna transmission scheme [4]

* In the case of no spatial multiplexing there is at most a single trans port block in a TTI.

* In the case of spatial multiplexing, with transmission on multiple layers in parallel to the same terminal, there are two transport blocks within a TTI.

\subsubsection{Physical Multicast Channel (PMCH)}

This channel defines the physical layer structure to carry Multimedia Broadcast and Multicast Services (MBMS). However, MBMS are not included in the first release of LTE. The PMCH is designed for a singlefrequency network and it requires that the base stations transmit with tight time synchronization at the same modulated symbols. The PMCH is transmitted in specific dedicated to subframes where the PDSCH is not trans mitted.

\subsection{Control Channels}

Control occupy the first 1,2 , or 3 OFDM symbols in a subframe extending over the entire system bandwidth as shown in Fig. 4. In narrow band systems (less than $10 \mathrm{RBs}$ ), the control symbols can be increased to include the fourth OFDM symbol.

\subsubsection{Physical Downlink Control Channel (PDCCH)}

This is the channel used by the eNodeB to carry control information to the UE. It carries an ACK/NACK response to the uplink channel, but also transports format allocation, UL scheduling grant and resource allocation information for the UE. In the future, there may be a need for multiple control signals, downlink scheduling control, uplink scheduling control and probably power control that needs to be dedicated for each mobile. A physical control channel is transmitted on an aggregation of one or several control channel elements, where a control channel element corresponds to a set of resource elements. Multiple PDCCHs can be transmitted in a subframe [5].

\subsubsection{Physical Control Format Indicator Channel (PCFICH)}

This channel carries the Control Frame Indicator (CFI) which includes the number of OFDM symbols used for control channel transmission in each subframe (typically 1, 2, or 3). The 32-bit long CFI is mapped to 16 Resource Elements in the first OFDM symbol of each downlink frame using QPSK modulation.

\subsubsection{Physical Hybrid ARQ Indicator Channel (PHICH)}

The PHICH carries the Hybrid automatic repeat request (HARQ) ACK/NAK which indicates to the user equipment (UE) whether the eNodeB correctly received uplink user data carried on the PUSCH. Binary phase shift keying (BPSK) modulation is used with repetition factor of 3 for robustness.

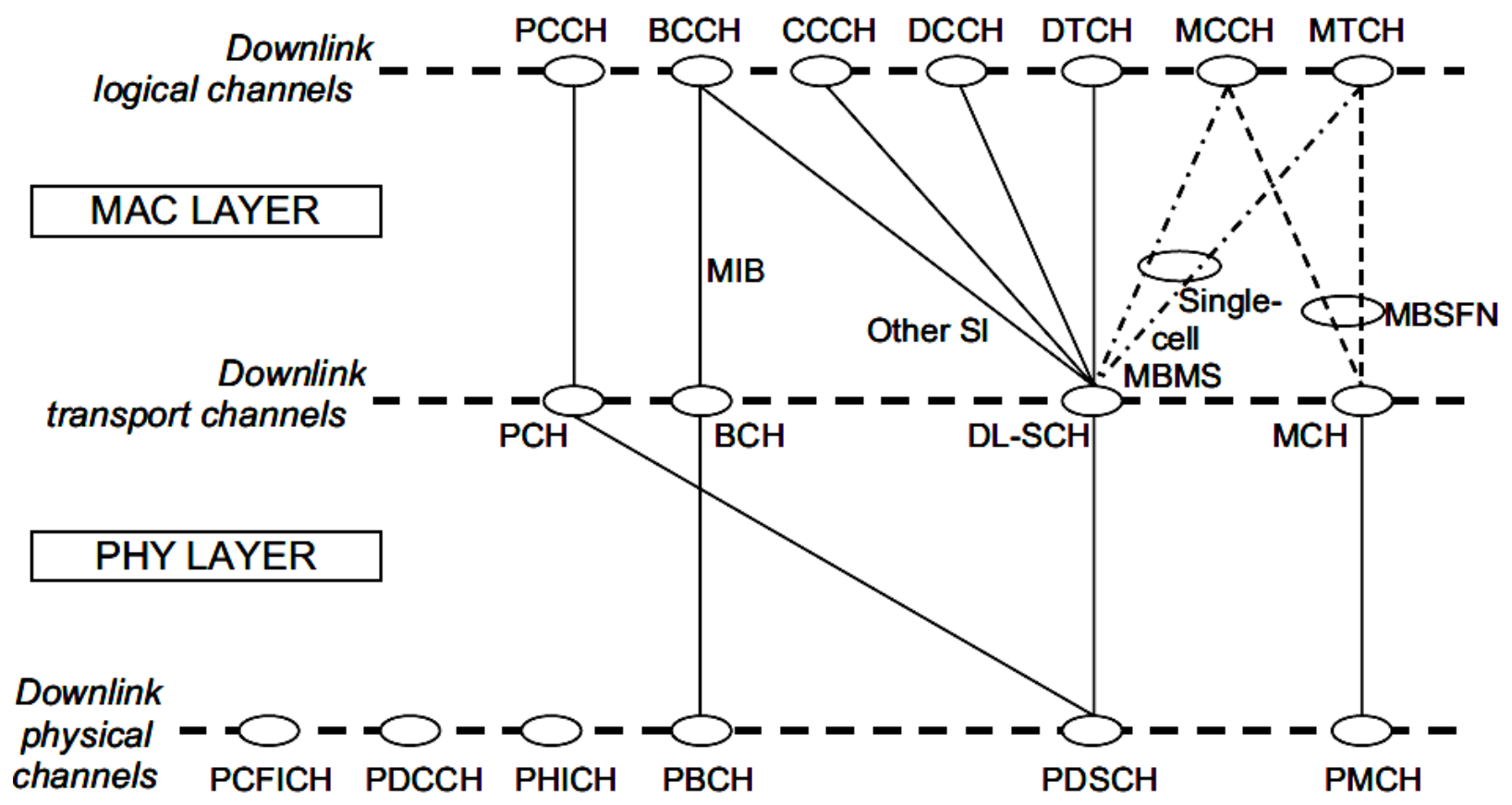

Fig. 4: Summary of downlink physical channels and mapping to higher layer 


\section{Uplink Physical Channels}

There are three physical layer channels defined for the uplink in LTE as described below:

\subsection{Physical Uplink Shared Channel (PUSCH)}

This channel carries user data. It supports QPSK and 16 QAM modulation with 64QAM being optional. Information bits are first channel-coded with a turbo code of mother rate of $1 / 3$ before being adapted by a rate matching process for a final suitable code rate. Adjacent data symbols are mapped to adjacent SCFDMA symbols in the time domain before being mapped across sub-carriers. After this interleaving process, bits are scrambled before modulation mapping, DFT-spreading, sub-carrier mapping and OFDM modulation. Channel coding is similar to that of the downlink.

The uplink scheduling interval is $1 \mathrm{~ms}$, similar to the downlink. However, it is possible to 'bundle' a group of 4 TTIs to improve performance at cell edge and reduce higher layer protocol overhead. In this case, a MAC PDU is segmented for transmission over multiple TTIs. In the frequency domain, transmissions are allocated based on multiples of $180 \mathrm{kHz}$ resource blocks. Uplink resources corresponding to the same set of sub-carriers are assigned for the two slots of a subframe. slot resulting in a frequency diversity gain and averages interference providing different hopping sequences are used in neighboring cells.

The PUSCH carries in addition to user data any control information necessary to decode the information such as transport format indicators and MIMO parameters. Control data is multiplexed with information data prior to DFT spreading. The PUSCH which carries data from the Uplink Shared Channel (UL-SCH), transport channel, uses DFT-Spread OFDM (DFT-S-OFDM). The transmitted processing chain is shown in Fig. 5. The information bits are first channelcoded with a turbo code of mother code rate $r=1 / 3$, which is adapted to a suitable final code rate by a ratematching process. This is followed by symbol-level channel interleaving which follows a simple 'timefirst' mapping [6]. In other words, adjacent data symbols end up being mapped first to adjacent SC-FDMA symbols in the time domain, and then across the subcarriers. The coded and interleaved bits are then scrambled by a length-31 Gold code prior to modulation mapping, DFT-spreading, subcarrier mapping and OFDM modulation. The signal is frequency-shifted by half a subcarrier prior to transmission, to avoid the distortion caused by the d.c. subcarrier being concentrated in one RB. The modulations supported are QPSK, 16QAM and 64QAM (the latter being only for the highest category of User Equipment (UE)) [7].

However, inter-slot frequency hopping is an option whereby different sub-carriers are used for the second
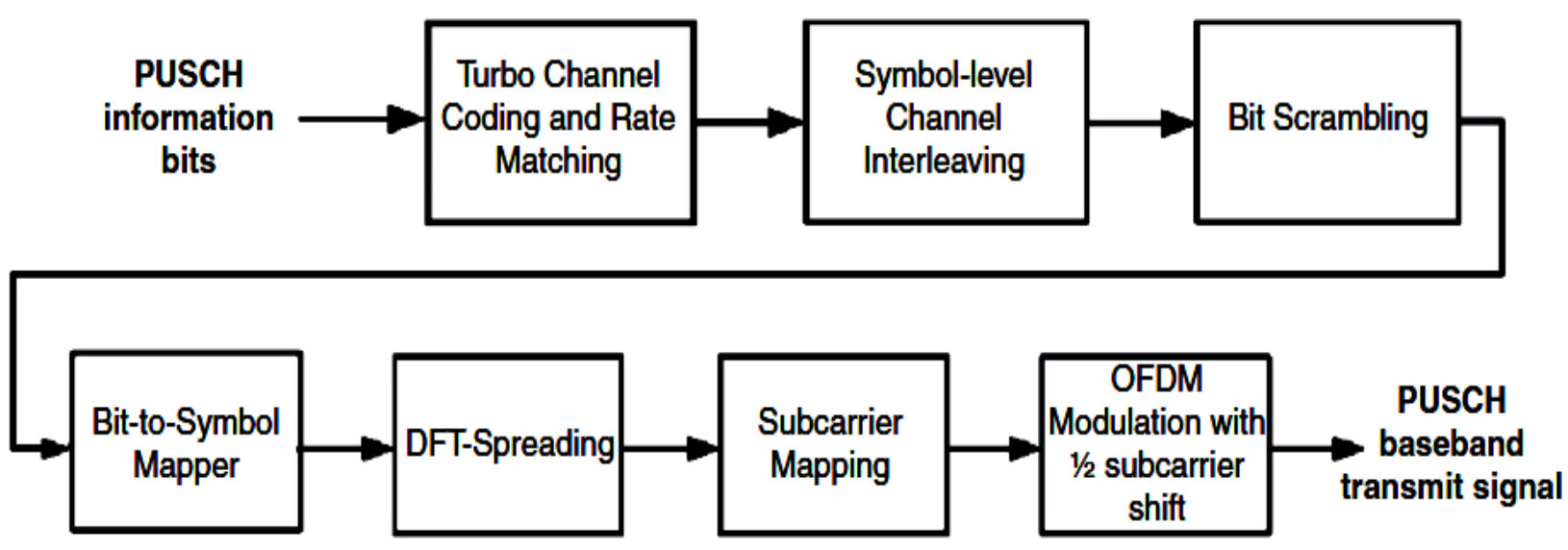

Fig. 5: Uplink physical data channel processing

Fig. 6 outlines the different steps of the UL-SCH physical-layer processing. Similar to the downlink, in the case of uplink carrier aggregation the different component carriers correspond to separate transport channels with separate physical-layer processing. Most of the uplink processing steps is outlined in Fig. 6 are identical to the corresponding steps for the downlink transport-channel processing.

The different steps for the uplink transport-channel processing are summarized below. 


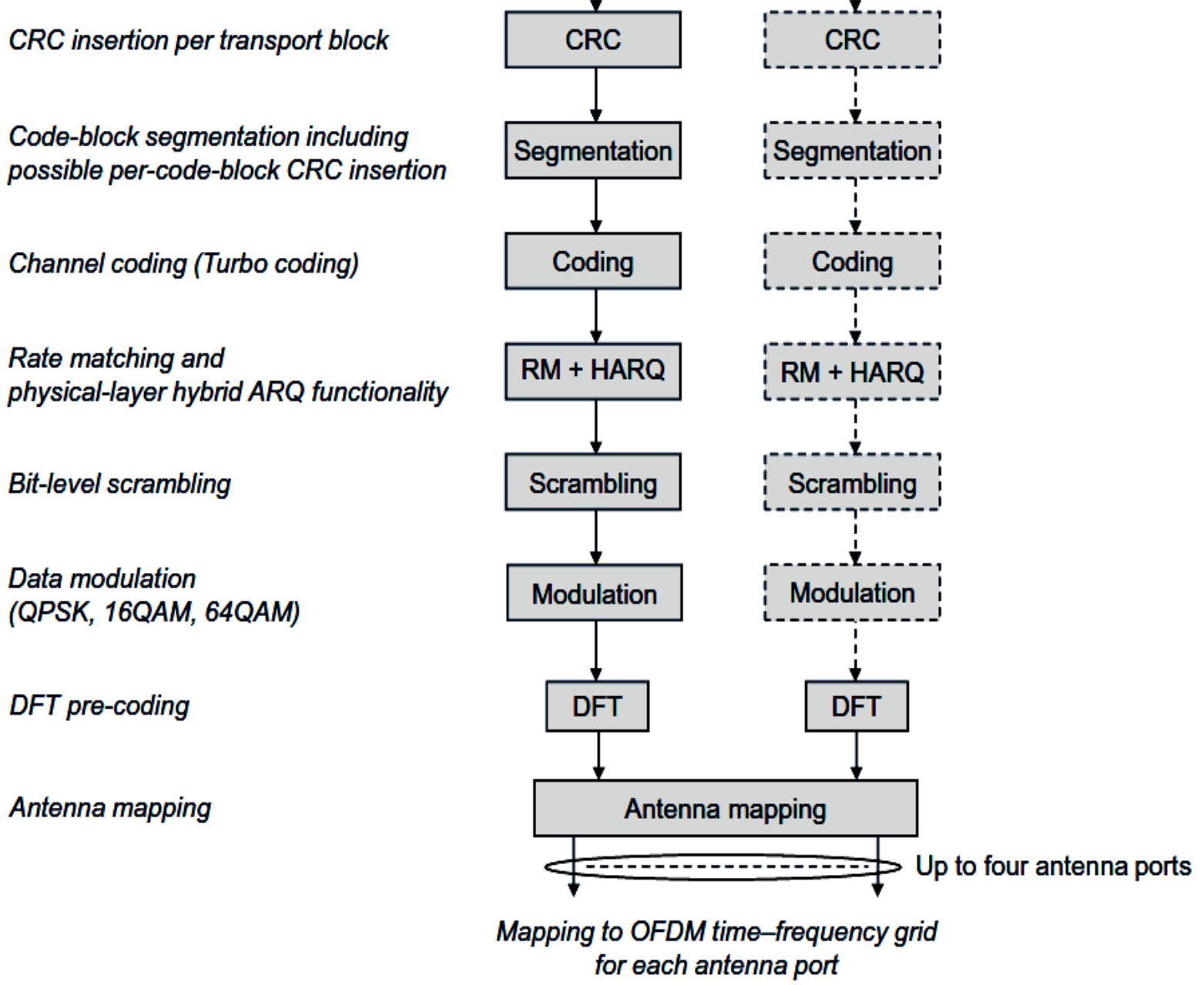

Fig. 6: Physical layer processing for UL-SCH [4]

\subsection{Physical Uplink Control Channel (PUCCH)}

Fig. 7 shows the logical split between different PUCCH formats and the way in which the PUCCH is configured in the LTE specifications [8]. The number of resource blocks in a slot reserved for PUCCH transmission is configured by the NHO RB -parameter. This broadcast-system parameter can be seen as the maximum number of resource blocks reserved for PUCCH while the actual PUCCH size changes dynamically based on PCFICH transmitted on downlink control channel. The parameter is used to define the frequency hopping $\mathrm{PUSCH}$ region. The number of resource blocks reserved for periodic Channel Quality Indicator (CQI) i.e., PUCCH Format $2 / 2 \mathrm{a} / 2 \mathrm{~b}$ is configured by another system parameter, N(2) RB [9].
$2 / 2 \mathrm{a} / 2 \mathrm{~b}$. However, with narrow system bandwidth options such as a $1.4 \mathrm{MHz}$ case, this would lead to unacceptably high PUCCH overhead [10]. Therefore, sharing the PUCCH resources block between Format $1 / 1 \mathrm{a} / 1 \mathrm{~b}$ and Format $2 / 2 \mathrm{a} / 2 \mathrm{~b}$ users is supported in the LTE specifications. The mixed resource block is configured by the broadcasted system parameter $N^{(1)}$ cs, which is the number of cyclic shifts reserved for PUCCH Format $1 / 1 \mathrm{a} / 1 \mathrm{~b}$ on the mixed PUCCH resource block.

Resources used for transmission of PUCCH formats $2 / 2 \mathrm{a} / 2 \mathrm{~b}$ are identified by a resource inde $\mathrm{n}(2) \mathrm{PUCCH}$, which is mapped directly into a single CS resource. This parameter is explicitly signaled via UE-specific higher layer signaling.

In general it makes sense to allocate separate PUCCH resource blocks for PUCCH Format 1/1a/1b and Format 
Resource blocks
reserved for PUCC

Format $2 / 2 \mathrm{a} / 2 \mathrm{~b}$, configured by $N_{\mathrm{RB}}^{(2)}$

Mixed resource block: contains both Format $1 / 1 \mathrm{a} / 1 \mathrm{~b}$ and Format $2 / 2 \mathrm{a} / 2 \mathrm{~b}$ resources Resource blocks
reserved for PUCCH
Format $1 / 1 \mathrm{a} / 1 \mathrm{~b}$

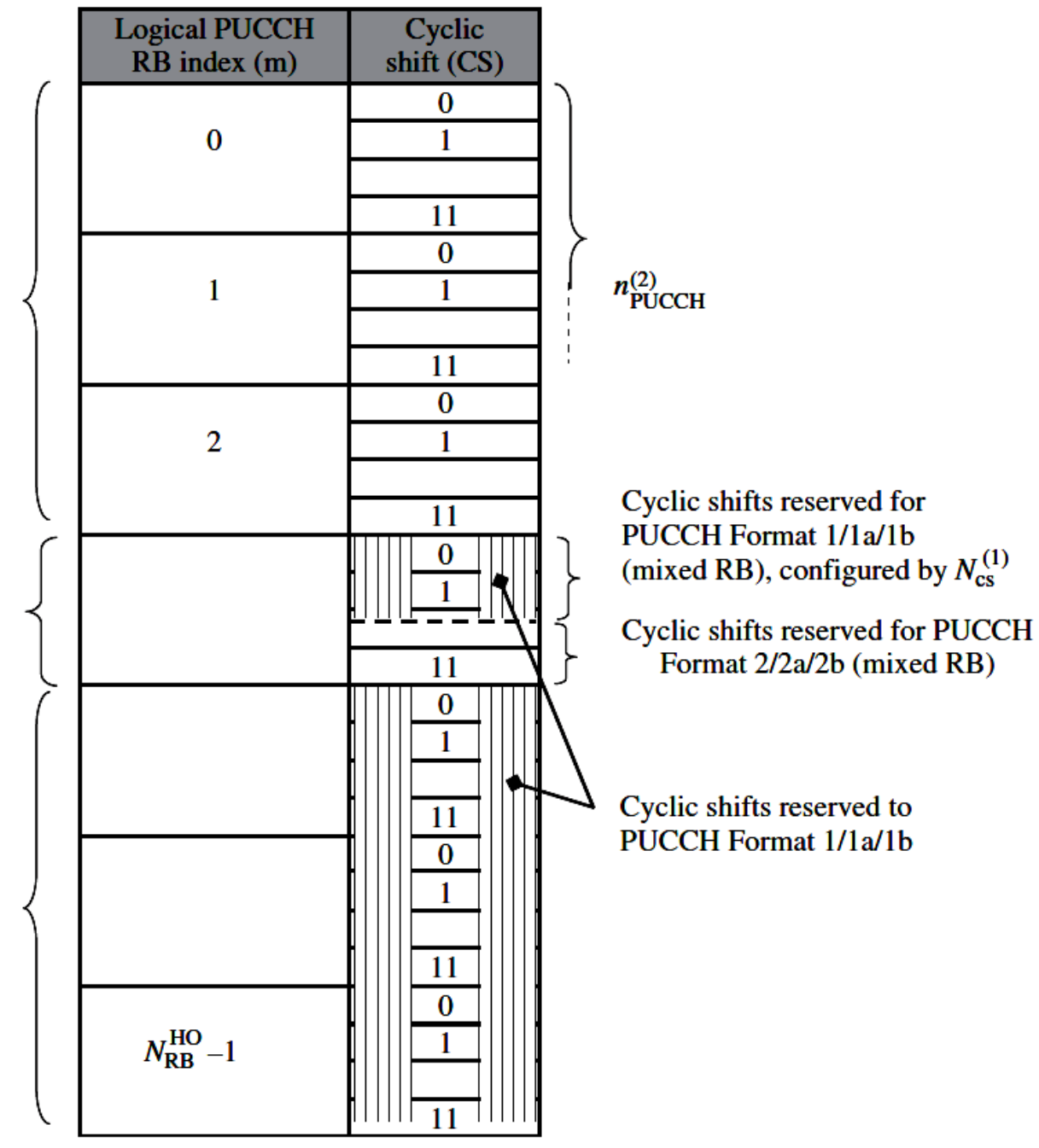

Fig. 7: PUCCH configuration

\subsection{Physical Random Access Channel (PRACH)}

Random access transmission is the only nonsynchronized transmission in the LTE uplink. Although the terminal synchronizes to the received downlink signal before transmitting on $\mathrm{RACH}$, it cannot determine its distance from the base station. Thus, timing uncertainty caused by two-way propagation delay remains on $\mathrm{RACH}$ transmissions. Appropriately designed Physical Random Access Channel (PRACH) occurs reasonably frequently, provides a sufficient number of random access opportunities, supports the desired cell ranges in terms of path loss and uplink timing uncertainty, and allows for sufficiently accurate timing estimation. Additionally, PRACH should be configurable to a wide range of scenarios, both for RACH load and physical environment. For example, LTE is required to support cell ranges up to $100 \mathrm{~km}$, which translates to $667 \mu$ s two-way propagation delay, as facilitated in the timing advance signaling range in the MAC layer [11].

In a LTE frame structure type 1 Frequency Division Duplex (FDD), only one PRACH resource can be configured into a subframe. The periodicity of PRACH resources can be scaled according to the expected RACH load, and PRACH resources can occur from every subframe to once in $20 \mathrm{~ms}$. PRACH transmission is composed of a preamble sequence and a preceding cyclic prefix with four different formats as shown in Fig. 8. Multiple preamble formats are required due to the wide range of environments. For example, the long CP in preamble formats 1 and 3 assists with large cell ranges in terms of increased timing uncertainty tolerance whereas repeated preamble sequences in formats 2 and 3 compensate for increased path loss. The guard period that is necessary after an unsynchronized preamble is not explicitly specified, but PRACH location in the subframe structure provides a sufficient guard period. Particular considerations are required only in very special cases. For each cell, 64 preamble sequences are configured and, thus, there are 64 random access opportunities per PRACH resource. PRACH occupies $1.08 \mathrm{MHz}$ bandwidth, which provides reasonable resolution for timing estimation. 
Format 0

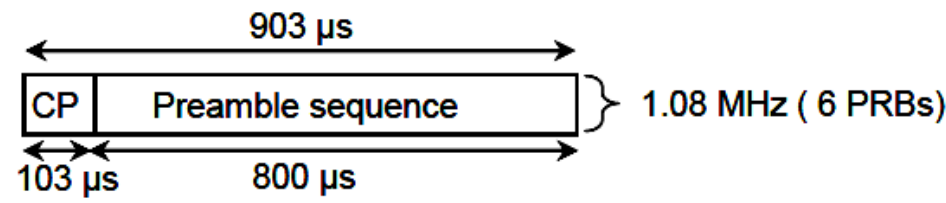

Format 1

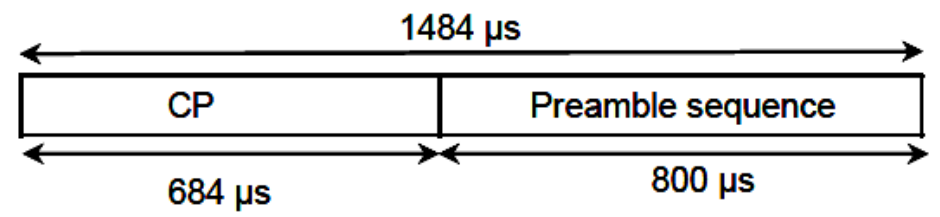

Format 2

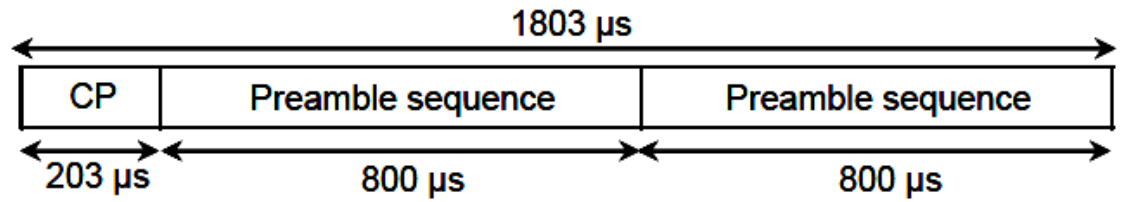

$2284 \mu \mathrm{s}$

Format 3

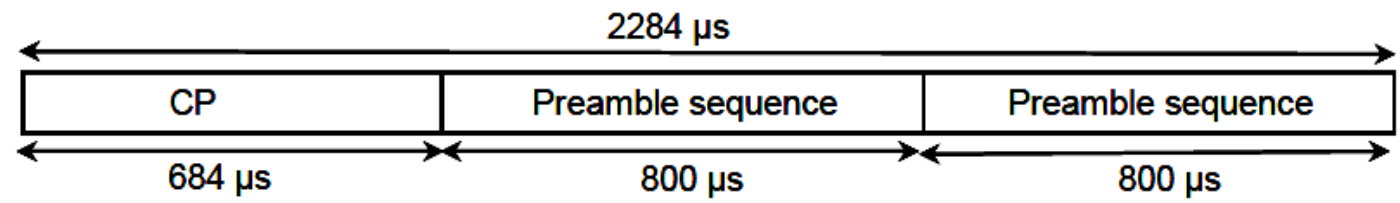

Fig. 8: LTE RACH preamble formats for FDD [11]

\section{Communication Channel Structure}

Each channel is characterized with a certain set of parameters and functions. There are logical channels that are mapped to transport channels. And, there are transport channels that are mapped to physical channels. Logical channels are identified with respect to the information carried by them and transport channels are distinguished according to their transmission characteristics. Similarly, physical channels are characterized by their configuration for data protection. Fig. 9 shows the mapping structure of channels for uplink and downlink [12].
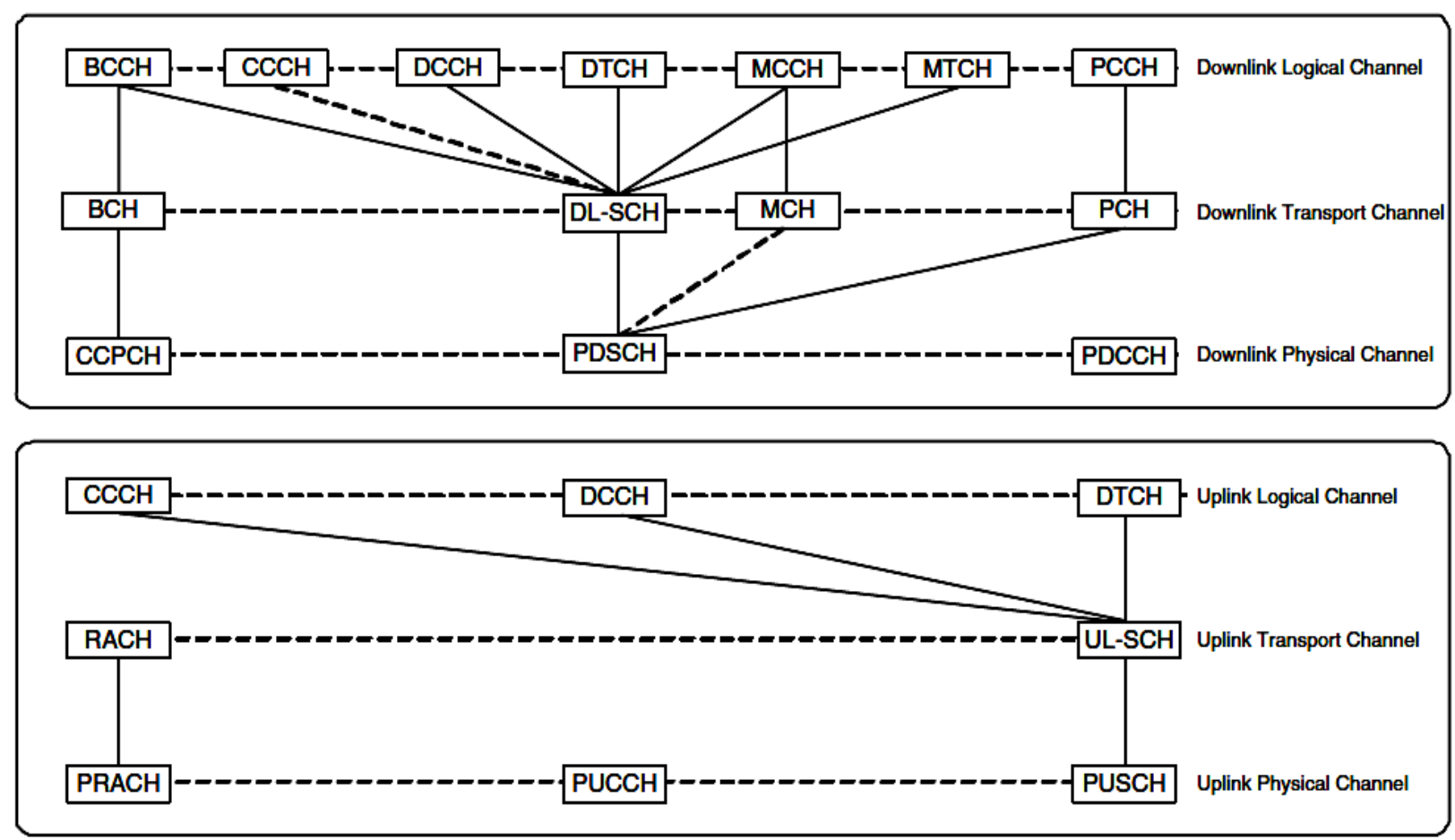

Fig. 9: Downlink and uplink channel mapping 
There are two types of logical channels: control and traffic channels. Control channels are as follows:

- $\boldsymbol{B C C H : ~ B r o a d c a s t ~ C o n t r o l ~ C h a n n e l ~ i s ~ t o ~}$ transmit broadcasting system control information.

- PCCH: Paging Control Channel is to transmit paging information when UE is unallocated.

- $\boldsymbol{C C C H : ~ C o m m o n ~ C o n t r o l ~ C h a n n e l ~ i s ~ u s e d ~ b y ~}$ UE when UE has no RRC connection.

- $\mathbf{M C C H}$ : Multicast Control Channel is used to transmit MBMS control information, which is point-to-multipoint (eNB to UEs) and only UEs that receive MBMS use it.

- DCCH: Dedicated Control Channel is a pointto-point bidirectional channel used by UE for RRC connection.

Traffic channels are as follows:

- DTCH: Dedicated Traffic Channel is a point-topoint bidirectional channel dedicated to one UE to transfer user information.

- MTCH: Multicast Traffic Channel is a point-tomultipoint channel for transmitting traffic data from the network to the UE

Transport channels provide structure passing data to/from higher layers, mechanism to configure PHY, status indicators to higher layers (CQI, error, etc.) and higher-layer peer-to-peer signaling. Transport channels for downlink are as follows:

- $\quad \boldsymbol{B C H}$ : Broadcast Channel transmits entire cell area with fixed transport format.

- DL-SCH: Down link Shared Channel is used for HARQ, dynamic link adaptation, UE DRX (discontinuous receive) for power save, dynamic and semi static allocation, and beamforming.

- PCH: Paging Channel is used for UE DRX, broadcast overcell coverage.

- $\boldsymbol{M C H}$ : Multicast Channel provides support for Multicast Broadcast -Single Frequency Network (MB-SFN) with semi-static resource allocation.

Transport channels for uplink are as follows:

- UL-SCH: Uplink Shared Channel is for HARQ, dynamic link adaptation, support for UE DRX, and dynamic and semi static resource allocation with $1 / 3$ turbo coding.

- $\quad \boldsymbol{R} \boldsymbol{A C H}$ : Random Access Channel is for limited control information, which has collision risk.
Transport channels are connected to physical channels. LTE downlink physical channels are as follows:

- PDSCH: Physical Downlink Shared Channel is utilized for data and multimedia transport. It is designed for high data rates with QPSK, 16QAM, and 64QAM modulation with 1/3 turbo coding and spatial multiplexing.

- PDCCH: Physical Down link Control Channel conveys UE-specific information with QPSKonly modulation for robustness. Up to first three OFDM symbols in the first slot of a subframe are used for PDCCH.

- CСPCH: Common Control Physical Channel conveys cell information with QPSK-only modulation and convolutional coding. $\mathrm{CCPCH}$ is sent close to center frequency.

LTE uplink physical channels are the following:

- PUSCH: Physical Uplink Shared Channel is allocated subframe basis by the UL scheduler with QPSK, 16QAM, or 64QAM modulation.

- PUCCH: Physical Uplink Control Channel carries uplink control information including CQI, ACK/NACK, HARQ, and uplink scheduling requests.

Each physical channel has a defined algorith $m$ for bit scrambling, modulation, layer mapping, precoding, and scheduling. It is also, noted that layer mapping and precoding are applicable when MIMO mode is present [12].

\section{Conclusions}

The LTE physical layer implements a number of technologies to deliver on requirements for high data rates and spectral efficiency. Aside from capacity, the physical layer is structured to provide low latency. The $1 \mathrm{~ms}$ subframe duration provides low latency through small scheduling intervals while maintaining low overhead related to higher layer protocols. The PHY is also well designed to provide high cell-edge performance with specific features such as dynamic bandwidth allocation to users and the design of reference signals and control channels which take into account more challenging path loss and interference environment at the cell edge.

This paper discussed the main types of downlink which contains: Firstly $\mathrm{PBCH}$ - transmits the broadcast channel $\mathrm{BCH}$. It is sent over 72 central sub-carriers on symbols 0 to 3 of slot 1 , using QPSK modulation. While the second type is PCFICH-transmits the size of the PDCCH in OFDM symbols. PDCCH-specifies the 
transport channel format, allocates resources and HARQ information. The forth type is PHICHacknowledges UL transmissions. PDSCH or DL-SCH carries data to the UE (payload) and finally $\mathrm{PMCH}$ carries the downlink payload.

On the other hand the uplink has three main types: PRACH - contention-based random access channel, used to adjust the UEtiming. It occupies $72 \mathrm{sub}$-carriers (5 RB) by 2 slots deep. Once the random access is successful, the message is transmitted in the UL-SCH. PUCCH acknowledges DL transmissions, sends CQI (Channel Quality Indicator) data, MIMO feedback and makes requests for UL transmission sand the third type is PUSCH or UL-SCH carries the UE data (payload).

\section{References}

[1] 3GPP Technical Specifi cation, TS 36.211, "Evolved Universal Terrestrial Radio Access (EUTRA); Physical channels and modulation," 3GPP, v 8.4.0, Sep. 2008.

[2] 3GPP Technical Specification, TS 36.214, "Evolved Universal Terrestrial Radio Access (EUTRA); Physical layer measurements," 3GPP, v 8.4.0, Sep. 2008.

[3] L. Korowajczuk, "LTE, WiMAX and WLAN network design, optimization and performance analysis," John Wiley, USA, pp.447-448, 2011.

[4] E. Dahlman, S. Parkvall, and J. Sköld., "4G LTE/LTE-Advanced for Mobile Broadband," Elsevier, UK, pp.143-204, 2011.

[5] P. Lescuyer and T. Lucidarme, "Evolved Packet System (EPS) The LTE and SAE Evolution of $3 \mathrm{G}$ UMTS,” John Wiley, pp.124, 2008.

[6] Motorola, 'R1-072671: Uplink Channel Interleaving', www.3gpp.org, 3GPP TSG RAN WG1, meeting 49bis, Orlando, USA, June 2007.

[7] S. Sesia, I. Toufik and M. Baker, "LTE - the UMTS long term evolution from theory to practice", John Wiley, UK, pp. 344, 2011.

[8] 3GPP Technical Specification, TS 36.211, "Evolved Universal Terrestrial Radio Access (EUTRA); Physical channels and modulation,", 3GPP, v 8.4.0 Sep. 2008.

[9] H. Holma and A. Toskala, "LTE for UMTS evolution to LTE-Advanced," $2^{\text {nd }}$ edit, John Wiley, UK, pp.98-99, 2011.

[10] 3GPP Tdoc R1-080931, “ACK/NACK channelization for PRBs containing both ACK/NACK and CQI,", Nokia Siemens Networks, Nokia, Texas Instruments.

[11] H. Holma and A. Toskala, "LTE for UMTS OFDMA and SC-FDMA based radio access," John Wiley, UK, pp.109, 2009.
[12] M. Ergen, "Mobile Broadband Including WiMAX and LTE," Springer USA, pp.393-395, 2009.

\section{Bibliography of Authors}

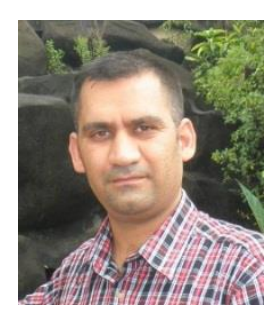

Aws Zuheer Yonis graduated from department of Computer Engineering at the Technical College in 2003 and completed his Master on Electrical and Electronic Telecommunication Engineering at University Tun Hussein Onn Malaysia (UTHM) in 2011. Currently he is studying doctorate of Telecommunication Engineering at UTHM from 2011. Since 2006, he became an engineer at college of electronic engineering at university of Mosul- Iraq. He has many publications in international journals and conferences. He is interested in wireless network, information security and mobile communication. He is a member of IAENG, SCIEI, SIE, CBEES, SDIWC, IACSIT, and Syndicate of Iraqi Engineering.

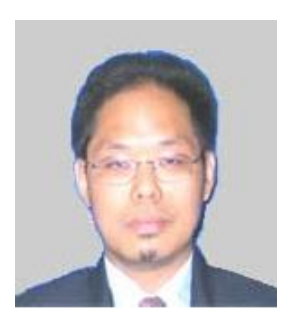

Mohammad Faiz Liew Abdullah received BSc (Hons) in Electrical Engineering (Communication) in 1997, Dip Education in 1999 and MEng by research in Optical Fiber Communication in 2000 from University of Technology Malaysia (UTM). He completed his $\mathrm{PhD}$ in August 2007 from The University of Warwick, United Kingdom in Wireless Optical Communication Engineering. He started his career as a lecturer at Polytechnic Seberang Prai (PSP) in 1999 and was transferred to UTHM in 2000 (formerly known as PLSP). At present he is a senior lecturer in the Department of Communication Engineering, Faculty of Electrical \& Electronic Engineering, University Tun Hussein Onn Malaysia (UTHM). He had 12 years' experience of teaching in higher education, which involved the subject Optical Fiber Communication, Advanced Optical Communication, Advanced Digital Signal Processing and etc. His research area of interest are wireless and optical communication and robotic in communication.

How to cite this paper: A. Z. Yonis, M. F. L. Abdullah,"Downlink and Uplink Physical Channels in Long Term Evolution", International Journal of Information Technology and Computer Science(IJITCS), vol.4, no.11, pp.1-10, 2012. DOI: 10.5815/ijitcs.2012.11.01 